\title{
Human-Induced Effects on RSS Ranging Measurements for Cooperative Positioning
}

\author{
Francescantonio Della Rosa, ${ }^{1}$ Mauro Pelosi, ${ }^{2}$ and Jari Nurmi ${ }^{1}$ \\ ${ }^{1}$ Department of Computer Systems, Tampere University of Technology, Tampere, Finland \\ ${ }^{2}$ Section of Antennas, Propagation and Radio Networking (APNet), Department of Electronic Systems, \\ Faculty of Engineering and Science, Aalborg University, Aalborg, Denmark
}

Correspondence should be addressed to Francescantonio Della Rosa, francescantonio.dellarosa@tut.fi

Received 1 June 2012; Revised 12 August 2012; Accepted 25 September 2012

Academic Editor: Stephan Sand

Copyright (C) 2012 Francescantonio Della Rosa et al. This is an open access article distributed under the Creative Commons Attribution License, which permits unrestricted use, distribution, and reproduction in any medium, provided the original work is properly cited.

\begin{abstract}
We present experimental evaluations of human-induced perturbations on received-signal-strength-(RSS-) based ranging measurements for cooperative mobile positioning. To the best of our knowledge, this work is the first attempt to gain insight and understand the impact of both body loss and hand grip on the RSS for enhancing proximity measurements among neighbouring devices in cooperative scenarios. Our main contribution is represented by experimental investigations. Analysis of the errors introduced in the distance estimation using path-loss-based methods has been carried out. Moreover, the exploitation of human-induced perturbations for enhancing the final positioning accuracy through cooperative schemes has been assessed. It has been proved that the effect of cooperation is very limited if human factors are not taken into account when performing experimental activities.
\end{abstract}

\section{Introduction}

With the advent of positioning systems, especially those relying on received signal strength (RSS), it became understood that the accuracy of location estimations is highly affected by the surrounding environment. A plethora of studies concerning presumed accurate solutions show custom coarse experimental activities, with limited repeatability for performance comparisons.

Additionally, the indoor environment sets great, although interesting, challenges for location-based applications since its intrinsic complexity severely affects the accuracy of measurements, causing huge signal fluctuations $[1,2]$. Overlapping channels, shadowing, multipath, objects, and sensitivity variations of heterogeneous wireless cards make it difficult to perform accurate applications targeting positioning services [2-6].

Alternatively to traditional approaches [7], new branches of positioning methods and techniques have been developed under the name of Cooperative Mobile Positioning [4]. The exploitation of the most reliable RSS measurements detected in the ad hoc links represent a valid and complementary approach to traditional noncooperative methods. The hybrid cooperative mobile positioning with signals of opportunity is deeply rooted in the exploitation of the most likely reliable short-range RSS measurements coming from neighbouring devices $[4,5]$. It also depends on the estimation of the distances among them (ranging), and processing data-fusion using nonlinear filtering, enhancing indeed the final position estimation with respect to conventional non-cooperative schemes [4].

The human body represents an additional source of inaccuracies causing unpredictable fluctuations in the RSS. The human body contains around $70 \%$ water, which absorbs part of the $2.4 \mathrm{GHz}$ WLAN radio signal causing a significant decay in the signal amplitude. In particular, the direction of the user body has attracted the research interest and has been identified as source of errors in the location estimation $[3,8]$. This is mainly because in one orientation there is a direct propagation path between the WLAN access point (AP) and the mobile station (MS) held by the user, while in the opposite orientation the user's body is obstructing the path in line-of-sight (LOS). For instance, experimental results in [8] indicate that the signal strength at a given location varies by up to $5 \mathrm{dBm}$ depending on the direction that the user is facing. Hence, the body of the user creates 
a systematic source of error and introduces a constant bias in the estimated locations that, if correctly accounted, can offer a beneficial impact on the positioning accuracy. Moreover mobile devices are held by the users, adding another source of errors for the RSS measurements: the hand grip [3]. The recent fervor on the iPhone 4 antenna-gate has contributed to surface in layman terms to the general public how important is the influence of the hand, due to the close proximity, showing an impact greater than the rest of the body.

In this paper, we demonstrate that human-induced errors cannot be ignored when performing experimental activities. In fact we show that both hand-grip and body-loss effects highly compromise even the undisputed benefits of cooperative schemes, highly corrupting the expected accuracy enhancements. We also experimentally demonstrate that although the effects of hand grip and body loss generate systematic errors, if correctly accounted and cognitively exploited, rather than roughly discarded or mitigated, it is possible to enhance the beneficial effects of the cooperation among devices in terms of positioning accuracy. To the best of our knowledge, this work is the first attempt to gain insight and understand the impact of both body loss and hand grip on the RSS for enhancing ranging measurements among neighbouring devices in cooperative scenarios. Our main contribution is represented by experimental investigations on ranging estimations, analysis of the errors introduced in the distance estimation using path-loss-based methods and exploitation of human-induced perturbations information for enhancing the final positioning accuracy of cooperative schemes.

The paper is organized as follows: Section 2 describes the conceptual flow from conventional to cooperative positioning lying the theoretical foundation for the subsequent experimental results shown in Section 3. Conclusions are finally presented in Section 4 .

\section{From Conventional to Cooperative Positioning}

2.1. Wireless Positioning. In wireless positioning different methods and techniques, based on signal of opportunity (SoO), have been proposed and adopted for experimental activities towards commercial location-based services [7] (Figure 1). Such methods can be divided into two main categories: mobile-based and network-based. While in the first the mobile station (MS) takes advantage of parameters gathered from base stations (BSs) or APs to determine its position, in the latter the BSs/APs measure parameters from signals coming from the MS, and the position calculation is performed on servers deployed in the network. In this section, we provide a brief overview of the main measurements and positioning techniques that can be used for positioning a MS using SoO.

2.1.1. Angle of Arrival ( $A O A)$. AOA makes use of multiarray antennas to estimate the angle of the line of arrival of the signal. The final position of the target MS is located at the intersection of the lines using triangulation. As antenna arrays are large in size, the positioning estimation is suitable

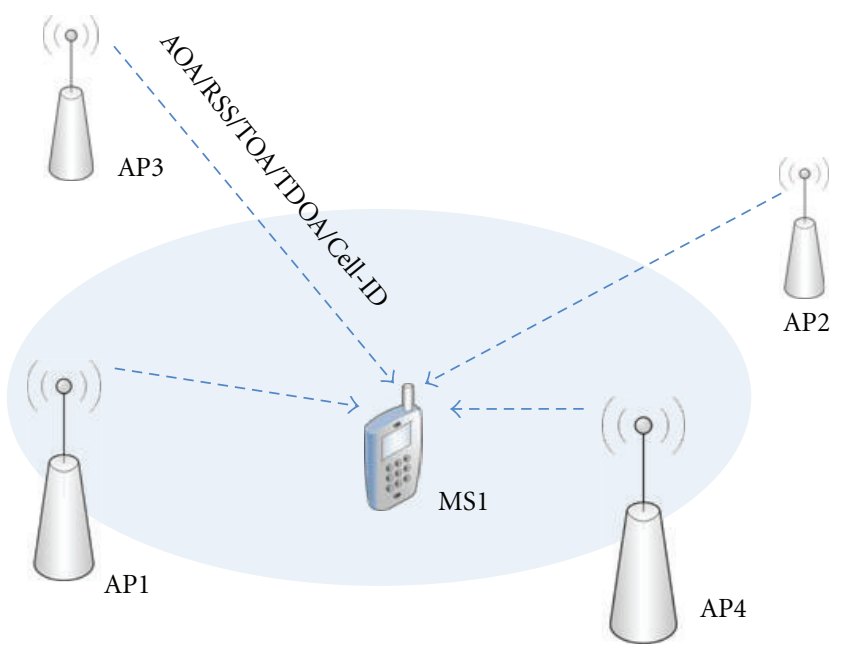

Figure 1: Wireless positioning.

to be performed by the network. AOA is mainly adopted for outdoor positioning [6], even though results on AOAbased positioning have also been performed indoors and reported in [6]. The direction of the arrival of the signals is highly corrupted by reflections and non-line-of-sight (NLOS) conditions deteriorating indeed the final accuracy.

2.1.2. Time of Arrival (TOA). TOA information can be retrieved by evaluating the time of arrival of the signal from MSs to fixed reference points with known coordinates [7] (GNSS satellites, BSs, APs, etc.) or vice versa. However, both entities need to be accurately synchronized in time. Once the distance between MS and at least three BSs is obtained from TOA, the final position estimation is then performed by using trilateration through the intersection of circles with radii determined from TOA measurements and centers at the known BS coordinates. Additional to synchronization problems, also reflections and non-line-of-sight (NLOS) conditions distort the TOA of the signals.

2.1.3. Time Difference of Arrival (TDOA). TDOA is based on evaluating the difference in the arrival times of signals from two different transmitters to receivers. TDOA values define hyperbolas between the two receivers on which the MS is potentially located. Positions of the MSs are then estimated at the intersection of the hyperbolas. [4]. Compared to TOA, the main advantage of this technique is that knowledge of the absolute time of the transmission is not needed as synchronization between transmitters and receivers is not required. Only synchronization among transmitters is required.

2.1.4. RSS. Positioning methods based on RSS estimate the MS location through theoretical, statistical, or empirical models to relate the strength of the received radio signal either to the distance between the BSs/APs and MS or to the MS location directly.

Parameters in the applied models are in principle experimentally determined in order to better adapt to the application environment. RSS-based positioning methods are 
divided into three main categories: cell identifier-based, fingerprinting, and pathloss-based. For mass market location-based applications, the RSS is considered more easily available than the aforementioned parameters as it can be passively listened from the APs of the infrastructure (e.g., WLAN). Technically the APs periodically broadcast beacon frames containing information for network identification (e.g., SSID, BSSID, RSS, RSSI) [3-7]. For network management and connectivity quality purposes MSs can switch through different channels and store information from any received beacon. The aforementioned process allows the MS to determine the cell identifiers and signal strengths of all visible APs.

(a) Cell ID. In the Cell identifier method, MSs perform passive scanning of the available radio channels (e.g., WLAN), and the position estimate is reported as the position of the relative BS (or AP) from which the strongest signal is received. With such method prior information about the locations of BSs/APs and their unique media access control (MAC) addresses or custom unique identifiers are required. The main characteristic of the method is the easy deployment and implementation despite its coarse accuracy level.

(b) Fingerprinting. Fingerprinting method is based on extensive and time-consuming experimentally built models relating recorded RSS values directly to the measured position. Models are obtained from off-line collected data from different locations sufficiently covering the area targeting positioning service $[1,6]$. For each location, a signal pattern is extracted and saved to the database of fingerprints with the location coordinates. In positioning phase, the on-thefly recorded set of RSS measurements in the coverage area are compared to the patterns stored in database and the position estimate is obtained from the pattern with the closest match with the measured RSS vector. Among all RSSbased methods, fingerprinting algorithms are considered robust against environmental impairments, as it makes use of location-dependent error characteristics of radio signals. The main disadvantage of fingerprinting is the tedious data collection phase as it is laborious and time consuming [6].

(c) Pathloss-Based. Pathloss models are conventionally adopted to convert RSS measurements into actual distances between the MS and BSs/APs. Once the aforementioned distances are estimated, trilateration can be adopted to estimate the position of the MS where at least three fixed reference points are needed. In indoor environments, multipath and attenuation caused by walls, other structures and even people complicate the modeling of signal propagation. Because of environmental impairments, the pathloss-based accuracy is typically worse than fingerprinting [6]. On the other hand, methods that utilize path-loss models to estimate distances are needed for fast and robust ranging measurements, like ad hoc WLAN connections between two MSs such as in cooperative positioning. Because of the low system set-up cost of pathloss-based positioning, and its better suitability for incorporating measurements from ad hoc connections,

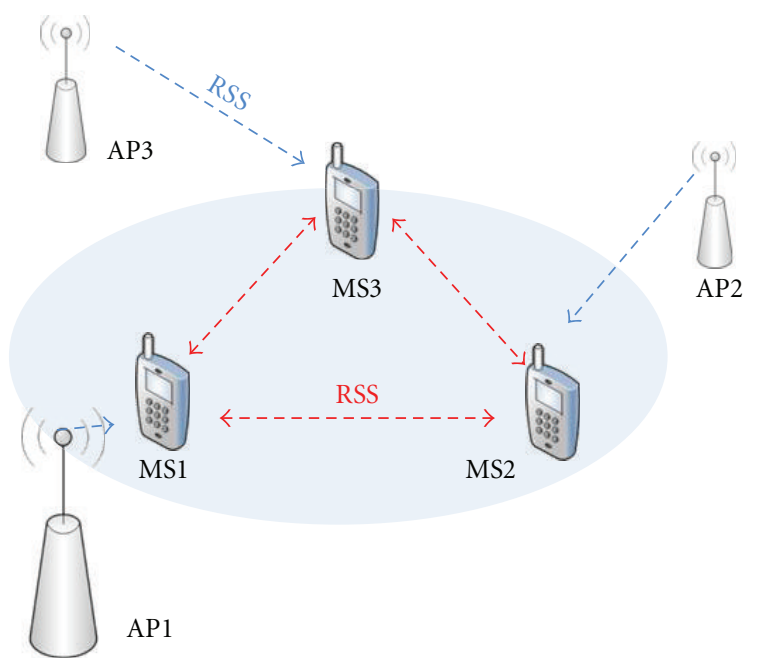

FIgURE 2: Cooperative scenario.

we concentrate on this technique by going deep into the main challenges encountered when building empirical pathloss models.

2.2. Cooperative Mobile Positioning. Cooperative mobile positioning (CMP) [4] uses hybrid schemes and data-fusion filters to combine short-range (MSs-MSs) and long-range (BSs/APs-MSs) measurements (Figure 2). The main concept is that "exploiting the most likely reliable short-range measurements coming from the neighboring mobile devices it is possible to enhance the location accuracy with respect to conventional techniques" $[4,5]$. In fact it has been demonstrated how the exploitation of spatial proximity estimated within a group of neighboring devices can enhance the location estimation accuracy $[4,6]$ and it can be easily applied in case of (i) outdoor environments, by merging the measurements from hybrid technologies; (ii) indoor environments, by combining infrastructure and ad hoc mode WLAN communications signals; (iii) GPS-equipped mobiles, where the location estimation can be enhanced in challenging environments [4]. The most promising approaches proposed in [4, 5] adopt least squares (LS), non-linear-least-squares (NLLS) algorithms and Bayesian filters. While Bayesian filters could be a potential alternative to the previous ones [4], the nonlinear characteristics of measurements and positions make the common Kalman filter (KF) not suitable for solving the proposed problem. Better results could be reached with extended Kalman filters (EKF), widely used for both positioning and tracking by linearizing the models and applying then the classical KF to the linearized system [4]. In this paper, we demonstrate results achieved by using an NLLS algorithm $[1,2]$ in the experimental activity with and without mitigating the effect of human-induced perturbations in RSS ranging measurements for the data-fusion algorithm adopted (NLLS). A fundamental step of cooperative mobile positioning is in the accurate ranging measurements based on the estimation of the close distances among peer neighbors connected in ad hoc mode. However, when the aforementioned distances are not properly and accurately estimated, the beneficial effects 
of cooperation are highly compromised. The effect of the hand grip and body loss not only corrupts long-range measurements (APs-MSs) but also short-range ones (MSs-MSs), hence degrading the potentials of a cooperative approach. The data-fusion algorithm, based on NLLS, is adopted. First RSS (APs-MSs link) are used to estimate the position of the MSs by using the LS algorithm [1]. The obtained estimates are the needed initial guesses for the NLLS when used in the non-cooperative case (conventional technique) (Figure 3(a)) [5]. Further details concerning the positioning algorithm can be found in $[4,5]$. To enhance the positioning accuracy (Figure 3(b)), RSS from the ad hoc MS-MS links are used to estimate the relative distances between the MSs and NLLS is used as data-fusion algorithm to combine all the measurements. Some notations need to be defined as follows:

$$
\begin{gathered}
\mathbf{X}^{[j]}=\left[x^{j} y^{j}\right]^{T}, \\
\hat{\mathbf{X}}^{(i)}=\left[\hat{x}^{(i)} \hat{y}^{(i)}\right]^{T}, \\
\hat{d}_{k}^{(i)[j]}=\sqrt{\left\{\hat{x}^{(i)}-\hat{x}^{[j]}\right\}^{T}\left\{\hat{x}^{(i)}-\hat{x}^{[j]}\right\}}, \\
\hat{d}_{k}^{(i)(j)}=\sqrt{\left\{\hat{x}^{(i)}-\hat{x}^{(j)}\right\}^{T}\left\{\hat{x}^{(i)}-\hat{x}^{(j)}\right\},}
\end{gathered}
$$

$\mathbf{X}^{[j]}$ represents the coordinates of $\mathrm{AP}_{j} ; \hat{\mathbf{X}}^{(i)}$ the estimated coordinates of $\mathrm{MS}_{i} ; \hat{d}_{k}^{(i)[j]}$ defines the estimated distance between $\mathrm{AP}_{j}$ and $\mathrm{MS}_{i}$ at iteration $k$ of the optimization routine when considering RSS from APs; $\hat{d}_{k}^{(i)(j)}$ represents the estimated distance between $\mathrm{MS}_{i}$ and $\mathrm{MS}_{i}$ at iteration $k$ of the optimization routine when considering RSS from ad-hoc links. The objective function to be minimized in order to determine the location of the MSs is described as follows $[4,5]$ :

$$
I\left(\hat{X}^{(i)}\right)=\sum_{i=1}^{n} \sum_{j=1}^{N}\left\{J_{T}\left(\hat{x}^{(i)}\right)\right\}^{2}+\sum_{i=1}^{n} \sum_{\substack{j=1, j \neq i}}^{N}\left\{J_{P}\left(\hat{x}^{(i)}\right)\right\}^{2},
$$

$n$ is the total number of MSs and $N$ is the total number of APs. The functions $J(\circ)$ can be defined as follows:

$$
\begin{aligned}
& J_{T}\left(\hat{x}^{(i)}\right)=\widehat{d}_{k}^{(i)[j]}, \\
& J_{P}\left(\hat{x}^{(i)}\right)=\hat{d}_{k}^{(i)(j)} .
\end{aligned}
$$

2.3. The Human Impact. The present section illustrates some cross-layer and cross-field topics often neglected in studies which focus on specific narrow areas without following holistic approaches that would result in a better comprehension of the underlying phenomena governing the application under investigation. In fact, there is often the selfish tendency of conducting experiments that do not result in providing a better understanding of the actual phenomena, mainly, because of their lack of generalization potential. A simplistic approach in designing experiments and carrying out measurement campaigns is undoubtedly a widespread

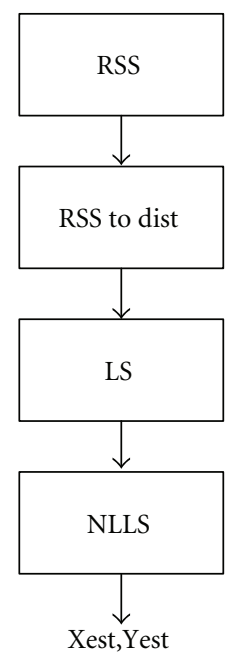

(a)

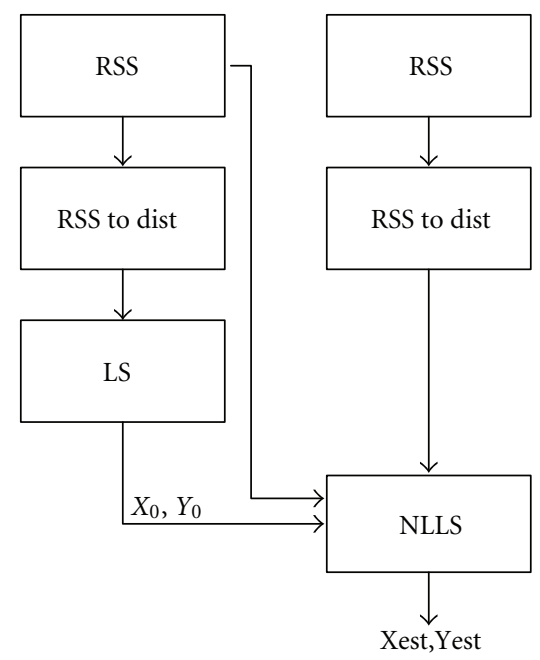

(b)
Figure 3: Data-fusion algorithms.

practice in most studies in literature. In fact, it is mandatory to take into account the most sensitive biases and properly identify beforehand the potential source of uncertainty [9]. As a result, the problem of oversimplification affects the understanding of channel behavior as well, which often does not consider at all the human-induced anomalies $[9,10]$. Unrealistic reference cases and optimistic assumptions might significantly deviate with respect to both-worst case scenario and average-use case. Though on one hand a certain degree of model simplification is always needed as a starting point, the subsequent investigations should tend to isolate the sources of uncertainty and trace back the particular sources of both errors and performance enhancement. Despite extensive research in localization over the past decade, quite little in proportion has been done to take into account the human-induced perturbations which users would experience through their smartphones in realistic environments that often are far away from the models presented in literature. The unpredictable temporal signal fluctuation arising from user interaction with any handheld device in the form of both signal blocking and motion in a multipath environment constitutes a major challenge for wireless communication performance. The user is an inescapable part of the wireless communication channel, and often it has not been included in models more because of oversimplification than gross lack of knowledge in the whole radiating mechanism. In addition to the regular and well-known dynamics in the propagation channel, mobile devices experience further dynamics in the antenna characteristic itself due to the often unavoidable and changing near-field interaction with users. Proper terminal design has to include not only the user interaction to yield good system performance on the overall wireless communication, but should integrate this information with the higher layers through a dedicated cross-layer design approach. Unlike simplistic static propagation channel assumptions, the user interaction itself is rarely static, having a further 
randomizing impact on the overall communication channel performances. The observed user-induced randomness and the underlying dynamics in the whole propagation channel highlight a strong challenge in designing effective localization systems, making it clear that it would be highly beneficial to put more effort in understanding the potential of userinduced anomalies cognitivity in cooperative localization, rather than focusing on the mere observation of the limits posed by an unfriendly propagation environment.

\subsubsection{Conventional Indoor Channel Modeling. The perfor-} mance optimization of cooperative localization algorithms requires dedicated efforts, such as the topics addressed in this section, as mobile terminals will often operate in far-fromideal wireless channel conditions. When modeling an indoor wireless channel, it is useful to break down the propagation mechanism as a superposition of different effects (expressed in logarithmic scale): channel $=$ pathloss + static shadowing + dynamic shadowing + fading.

2.3.2. Pathloss and Fading. The usual path-loss is simply defined as that portion of the propagation loss which depends uniquely on the distance between the access point and the terminal or between two terminals. However, for indoor-to-indoor links, the path loss is also dependent on the number of walls in between the transmitter and the receiver, having different weights for walls of different materials, such as thick brick walls and thin plasterboard walls. Small-scale variations of the signal power are accounted for in the fading part, which also depends on the speed of the mobile terminal.

2.3.3. Static and Dynamic Shadowing. Static shadowing is due to signal obstructions related to the shadowing effect of large objects which are time-invariant and it is also due to the emergence of destructive and constructive interference of coherent multipaths. Dynamic shadowing is the propagation portion reflecting the well-described large-scale variations around the pathloss, which typically follow a log-normal distribution and may be correlated between different links.

2.3.4. Channel in Presence of the User. The operating environment of wireless communications in proximity with the user's body is quite different from more traditional wireless networks, as one end of the link is affected by the fact that the body is in the near field of the device, dictating the nonstationary behavior of the channel. Proximity of a wireless device to external objects such as user's body, tinted doors, and metallic doors and strongly affects the propagation environment. Nevertheless, most of the propagation phenomena happen away from the body in the surrounding space, allowing the usage of the aforementioned well-established propagation models. The reliability of a wireless link operating in such an environment is strongly conditioned by the user's body influence, whose impact on propagation demands a rigorous analysis. There are a series of time-varying conditions, such as user movement, orientation, hand grip, and posture. Using a very simplistic approach, the human body can be modeled electromagnetically as a homogeneous dielectric cylinder, where impinging waves generate reflection and diffraction phenomena. The extent of these effects will depend on several factors such as the operating frequency, the relative dimensions of the body with respect to the wavelength, and the average composition of the human tissues. However, not all the body parts respond in the same way to the exposition to electromagnetic waves, as if one particular body part is not large with respect to the wavelength, it will have a negligible effect on the propagation mechanism and will yield small fluctuations. When we are dealing with wireless devices with low operating frequencies, their ground plane will result to be comparable with respect to the half a wavelength, becoming an integral part of the radiating structure. The presence of the user' body naturally leads to changes in the purity of the antenna radiation pattern and input impedance, causing a corresponding reduction of the total efficiency. Antennas in common devices are very seldom directional, as they have to cope with a rich multipath environment. In fact there is no sense in designing a directional antenna that would be immediately corrupted by the effect of the user's head and hand. When a standard wireless device rotates around a blocking object such as the user's body, its radiation pattern is affected in such a way to emulate the behavior of a directional antenna. Moreover, the presence of a large object in between the wireless links causes a consistent drop in the RSS. Though such a drop is typically the strongest when the receiving device is totally in the shadow area of the user's body, it might also happen that because of the multipath effect there is a signal drop of different entity. By using the RSS measured at one or multiple locations, it is possible to give a first estimation of the distance between the transmitter and the receiver making basic assumptions on the propagation model. However they end up in being inaccurate in reflecting the user-induced anomalies in a practical environment. In fact, the idealized simple assumption that RSS decreases as the receiver moves away from the transmitter often breaks down in practice and spoils even the beneficial cooperation potential in localization. An important matter is also the choice of the maximum physical radius within which human-induced disturbances may be considered to be an integral part of the antenna. The human body is nothing else but a lossy dielectric load for the antenna system point of view [11-15]. Beside health hazard speculations, electromagnetic waves naturally tend to dissipate in our bodies by Joule effect. In fact it is more relevant to focus on the influence of the body on the antenna rather than the contrary [11]. This has several effects on the antenna system, such as lowering of the total antenna efficiency, radiation pattern deterioration, and throughput reduction [11].

2.3.5. Statistical Antenna Modeling. There is an undisputable need for simple but representative channel models in such a way of being able to account for the variability of the propagation in a parameterized manner. This can be achieved either by using advanced models or by properly extracting statistical models from ad hoc databases. Even though it is possible to describe antennas in a deterministic way, their performance in the presence of the user has an inherently stochastic nature. In [16], a model is presented for the stochastic signal variations in the presence of the user's body, 
assuming as a first approximation a normal distribution and being able to quantify both mean and standard deviations. It was found that for a typical WiFi signal, the standard deviation in the received signal may reach up to $2 \mathrm{~dB}$ when only the user's torso is considered. However, as stated in several publications, the effect of the hand is typically more significant than the rest of the body, adding further sources of randomness in the link budget determination. Only recently the first attempts to statistically model the antenna system close-by environment are being found in the literature [12], recognizing that the influence of the human body hides a substantial fraction of the link budget [13]. In fact, when a link budget is typically defined from the overall system perspective $[14,15,17-21]$, there is always a lack of real usage figures of merit and typical values tend to be collected referencing papers that might not be appropriated and pertinent anymore due to the actual changing conditions. One of the best examples is the $3 \mathrm{~dB}$ body-loss figure recommended for GSM, while it is always $10 \mathrm{~dB}$ or more in real usage and even 20-25 dB in low-end mobile phones [14]. This misleading approach is for instance one of the main reasons for problems in DVB-H, which has almost no indoor coverage support and suffers because of the influence of the hand $[15,21-25]$.

2.3.6. The Superantenna Concept. The user interaction with the device is condensed in a single propagation aspect, so that the rest of propagation can be seen as a superposition of easily identifiable combinations [16, 25-28] of propagation parts, ensuring the practical applicability of the aforementioned statistical antenna modeling. In [29-31], the user's body is considered as an integral part of the handheld device, leading to the concept of super-antenna. This makes it possible to extend the composite channel approach to include user effects by combining the channel with the radiation patterns of the super-antenna.

2.3.7. The Hand-Grip Effect. The original sin of many mobile device manufacturers has been the fact of overlooking the importance of the hand grip on the communication performance. Usually usability studies are performed on a limited number of users that might not fully capture the exact complexity of real usage patterns [9]. Several studies are mainly focused on ergonomics and operability, while only recently we have witnessed a growing awareness of the user's influence on the radiating performance [17]. The hand's influence, due to the close proximity, is greater than the rest of the body [21], and the overall variation of the RSS is very sensitive also to the relative position of individual fingers [17, 18]. Though very often literature studies present exceptional performances, however common practice tells us that if all the real-life conditions are correctly accounted for most proposed schemes it might even be worse than the reference case. The recent fervor on the iPhone 4 antenna-gate has contributed to surface in layman terms to the general public how important is the interaction between the user and the mobile device. Only recently the first hand phantoms have been standardized and investigated by CTIA and COST2100 $[20,21]$. However, even using standardized hand phantoms does not solve all problems, as manufacturers might tend to optimize the mobile phones only for passing a very specific certification tests. This means that not only proper mobile phone design should take into account the real usage variability, but any successful service which relies on sensing link quality-related quantities must properly include the effect of the hand.

2.3.8. Cognitive Design. Harnessing the influence of the human body on the overall measurements and performances of mobile phones can bring unexpected advantages [22]. By knowing statistically what is the most likely grip for a mobile phone it is possible to realize more robust antenna designs [22]. Moreover, the growing number of sensors on mobile phones and the upcoming cognitivity in smartphones can provide additional information that can ease communication and measurements analysis and even pave the way for new features [23]. Adaptive impedance matching and capacitive sensing of the position of the fingers of the user [24, 25] has, for example, demonstrated to improve the antenna efficiency by compensating both mismatch and absorption loss in real products. This can also be used in localization algorithms that would be able to filter out the aforementioned effect of the near-field in the RSS depending on what the usage pattern is [26]. Thus, there are still many exciting research opportunities ahead on compact antenna systems in a radio channel context for the years to come from which positioning would really benefit in terms of reliability and accuracy of measurements.

2.4. Related Works Concerning Human Body Impact. Considering research on localization, the user's impact on RSS measurements is shown to yield a $67 \%$ accuracy degradation [8] in the median error when the neighbour technique is considered and the user's body blocks the signal between the APs and the MSs. Moreover, other authors show that a human body may corrupt the RSS up to $3.5 \mathrm{dBm}$ and that exists a strong correlation between the measured RSS and the direction that the user is facing. In fact it is observed that omnidirectional signals are given directional properties because of the presence of the user's body, where usually the statistical distribution of the RSS varies more than the mean [30]. The effect of the user's presence on the RSS distribution is shown in [30], where a large spread of the RSS range is reported, that is, a standard deviation increase from $0.6 \mathrm{dBm}$ to $3.0 \mathrm{dBm}$ and a mean value change from $-70.4 \mathrm{dBm}$ to $-71.6 \mathrm{dBm}$. However, it is not clear if the user's body is really responsible for blocking the propagation path from the AP or not. By using samples collected in four different directions and LOS and NLOS paths between the mobile device and the WLAN AP, the effect of user's orientation was also studied in [30]. In case of NLOS, the signal coming from the AP had an attenuation of $6.0 \mathrm{dBm}$ between the highest and the lowest RSS levels, while for the LOS case a $10.0 \mathrm{dBm}$ loss was observed in the user's body obstruction direction. Even worse, if the user turned to the opposite direction, another AP having low RSS values when the user was directly facing it was completely undetectable. The performance of positioning methods that exploit RSS measurements may be severely degraded by 
the aforementioned effects. As the exact number and sizes of humans in a building at any time is generally a finite but random number [8], it is virtually impossible to characterize the variable signal loss precisely as far as pathloss modelbased methods are concerned. Fortunately, in fingerprintbased methods the effect of the user's body can be taken into account explicitly by extending the radio map and including fingerprints collected at each location while the user's body is facing at different directions. As an example, in [8] it is also recorded the direction $(d)$ — north, south, east, or westin addition to the user's location $(x, y)$. However, in the aforementioned paper the information on the direction is only used to assess the effect on the positioning error of a wrong direction assumption. Likewise, the RSS fingerprints at each location in two opposing directions were collected [32-35]. The accuracy performance could be enhanced if the direction of the user's body during positioning was known or could be implied somehow. At this purpose, we may mention [31] where low cost and low power digital compasses are used to detect the user's orientation, so that only those fingerprints collected during the offline phase that have a similar orientation are selected in determining the user's position. The positioning accuracy has been recently shown to greatly improve using the digital compass integrated into the Google Nexus One smart phone [36]. Another solution to mitigate the user's body effect is to exploit novel antenna designs, for example, omnidirectional wearable antenna, as suggested in [8]; this would be applicable to model-based methods as well. There is a general agreement that the impact of user's orientation consists in a significant signal loss in the LOS direction that the user's body is blocking. However, holding the mobile device in different orientations, for example, vertically or horizontally, while the user is static, is an ulterior case that has not been studied in the literature before. The relative location of the antenna with respect to the neighboring AP has an effect on the observed RSS values which depends on the orientation of the device. Moreover, the user may block the antenna unintentionally with his particular hand grip, causing additional signal attenuation and accuracy degradation (Figure 5) as reported in [3]. To the best of our knowledge, our work is the first genuine attempt to gain insight on the impact of mobile device's orientation, the body loss, and the hand grip on the RSS measurements in the localization of mass market devices in cooperative scenarios. Our main contribution is the on-thefield experimental analysis of the aforementioned parameters on the RSS samples. We then investigate the amount of error introduced in the distance estimation from the respective MSs that is due to these conditions showing in a test environment the impact on the final position estimation.

2.5. Human-Induced Effects on Cooperation. In this context, heterogeneous technologies and mobile terminals coexist and cooperate with the objective of helping each other for enhancing accuracy of their estimated positions. This can be accomplished by sharing link information with peer nodes connected in ad-hoc mode and exploiting their spatial diversity with advanced positioning algorithms [4]. Sharing radio signals that are just enough to ensure network connectivity among mobiles, the ad hoc network model achieves better performances over the stand-alone infrastructure one $[3,4]$. However, as the close-proximity [37] range estimation is the core of the proposed cooperative approach, it needs to be very accurate, which does not happen due to the effect of body loss and hand grip (Figure 6) in the RSS to distance estimations using path-loss models, as it will be demonstrated in Figures 14 and 15.

\section{Experimental Activity}

In this section we present the effect of the human body and the hand grip on RSS measurements for localization of mass market devices in indoor environments, demonstrating the strong influence they have in the evaluation of the RSS and distance estimations to be used in cooperative schemes. The experiment has been performed at Tampere University of Technology, Department of Computer Systems in an open area, with dimensions of $20 \times 20$ meters by using mass market devices. In particular we have adopted Nokia N900 Smart Phones for recording RSS from a total of four APs 802.11n Cisco.

3.1. Empirical Pathloss Model. Theoretically, a free-space propagation model could be used for converting RSS into distance [3]. However, the accuracy of measurements will decrease in environments where obstructions and other impairments are present. As mentioned before, indoor scenarios can be characterized by several objects (static or moving) producing reflections, diffraction, and scattering [37]. This means that the signal decay is affected not only by the distance between transmitters and receivers, but also by obstructions [3]. The theoretical model described in [2, 3] shows the large scale behavior of the propagation given by [6]:

$$
d=10^{\left(\left(P_{t x}-P_{r x}+G_{t x}+G_{r x}-X_{a}+20 \log _{10}(\lambda)-20 \log _{10}(4 \pi)\right) / 10 n\right)} .
$$

However, this model does not take into account any characteristics of the environment and it is not suitable at all for practical applications in real life. At this purpose the pathloss model adopted in this work has been obtained empirically (Figure 7) by following the procedure described in $[2,3]$, hence placing the MS at several distances from $1 \mathrm{~m}$ to $20 \mathrm{~m}$ respect to the AP (Figure 8 ), measuring the RSS, and storing the value with the highest number of occurrences at each step. Figure 8 shows the obtained model, which has been filtered with a 4 th order polynomial approximation.

3.2. Long-Range versus Short-Range Measurements. Figures 9 and 10 show the RSS measured in short-range, close proximity $(2 \mathrm{~m})$, and at long-range $(15 \mathrm{~m})$. As expected, measurements from $2 \mathrm{~m}$ are more reliable if compared to longrange ones as they appear to be more stable and less affected by fluctuations [3]. Being more reliable the short-range measurements offer the possibility to accurately estimate the distances between MSs deployed in the area. However, this is only true if measurements are performed without the effect of both hand grip and body loss, hence, if they are sufficiently stable or not corrupted. 


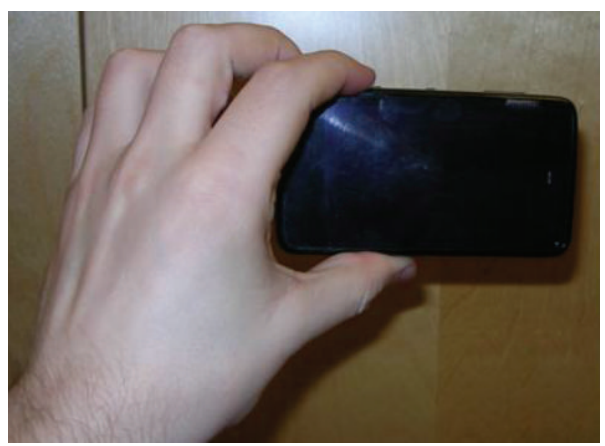

(a)

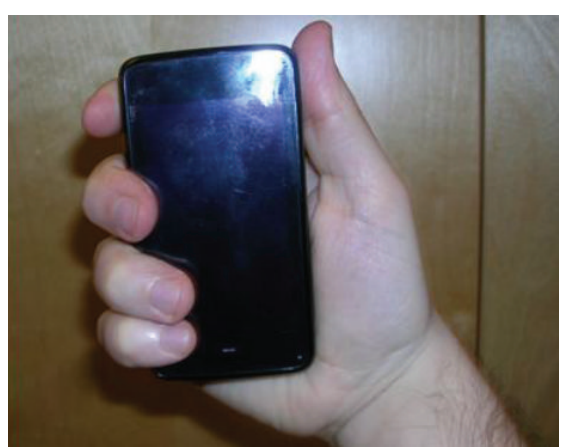

(b)

FIgURE 4: Hand-grip positions.

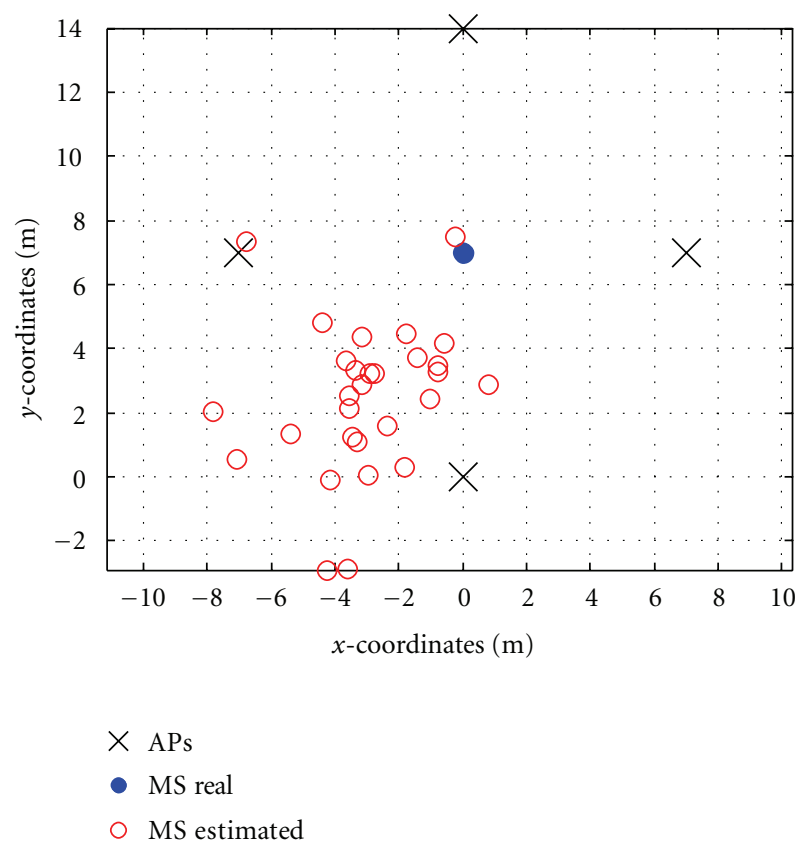

FIgURE 5: Human body impact on positioning estimation [3].

3.3. Device Orientation. Measurement campaigns with mass market devices are always a tricky task since devices' specifications are not always detailed and companies do not share technical secrets and performances capabilities. In addition to the traditional environmental factors to take into account [3] related to RSS, the orientation of the device with respect to the AP is one of them. Specifically, depending on the particular position respect to the AP, different values of RSS are measured. In [3], 32 positions have been defined. Hence devices report different RSS values depending on the specific orientation respect to the $\mathrm{AP}$ and it is straightforward to understand that by applying a RSS-to-distance conversion model $[3,6]$ the orientations of the device highly affect the distance estimation, consequently compromising the positioning estimation accuracy.

3.4. Body-Loss Effect. The human body [37] has a huge impact on the RSS measurements for localization purposes

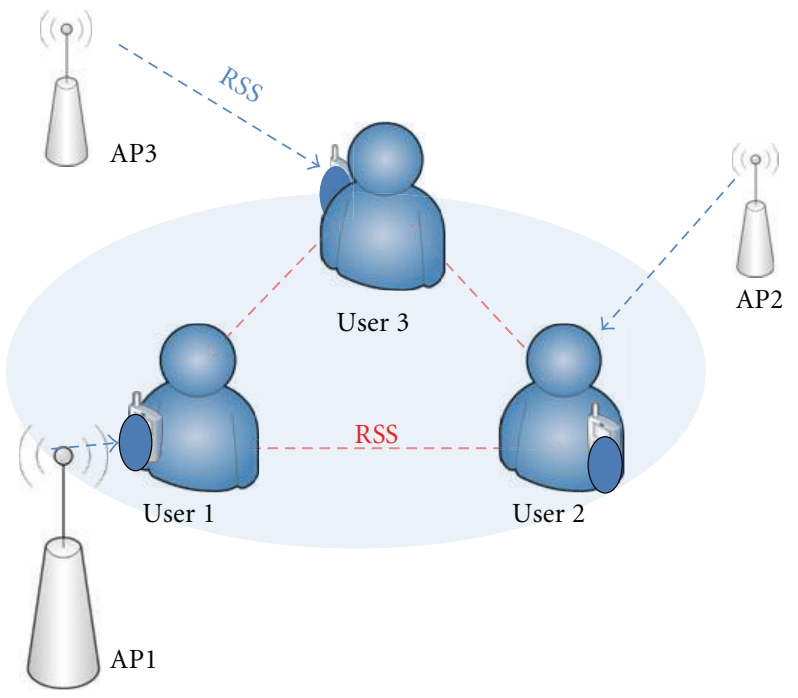

FIGURE 6: Cooperative positioning with human body effect.

[3]. Figure 11 shows the measured effect in four extreme cases: (a) (front) line-of-sight (LOS) between AP and MS; (b) (back) no LOS between AP and MS, with the user's body fully occluding the path; (c) (right) AP on the right side of the user; (d) (left) AP on the left side of the user. In this case the MS is placed at $3 \mathrm{~m}$ from the AP. Figures 11 and 12 show the real effect of the human body on the RSS measurements and estimated distances performed using the empirical pathloss model reported in Section 3.1. As expected, case (a) shows the highest RSS, while case (b) shows the lowest. Consequently, also the distance estimation from the AP is compromised as reported in Figure 12.

3.5. Hand-Grip Effect. The hand-grip effect experiment has been performed using the simple grips shown in Figures 4(a) and 4 (b) by maintaining the same orientation (a) as reported in Section 3.4. In Figure 4(a), the hand is not covering the WLAN antenna, while in Figure 4(b) the antenna is fully covered. The AP is placed 3 meters away from the MS under test and the recorded RSS with the estimated distances are reported in Figure 13 (squares) and Figure 14 (squares) 


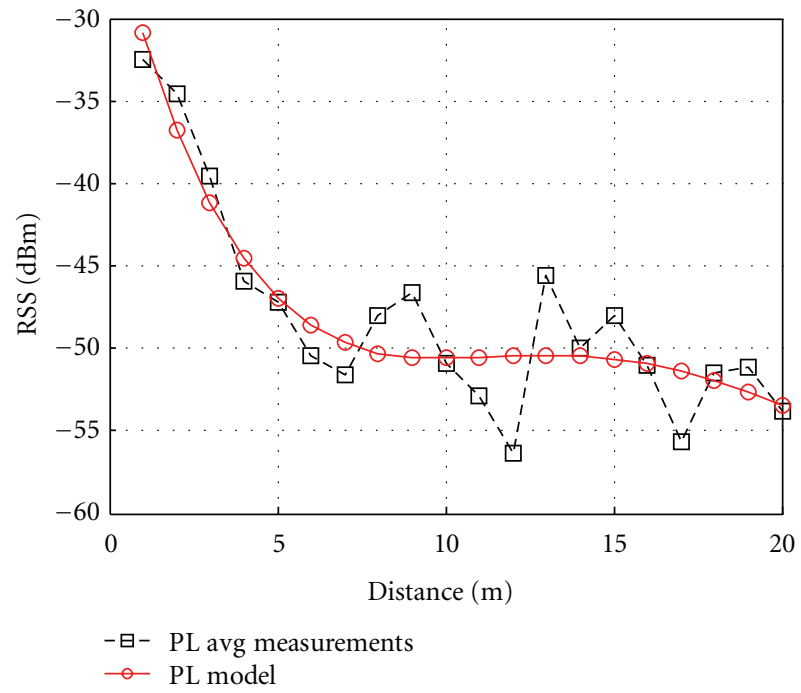

Figure 7: Empirical pathloss model.

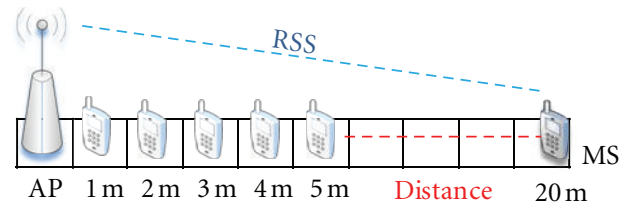

Figure 8: Pathloss model definition.

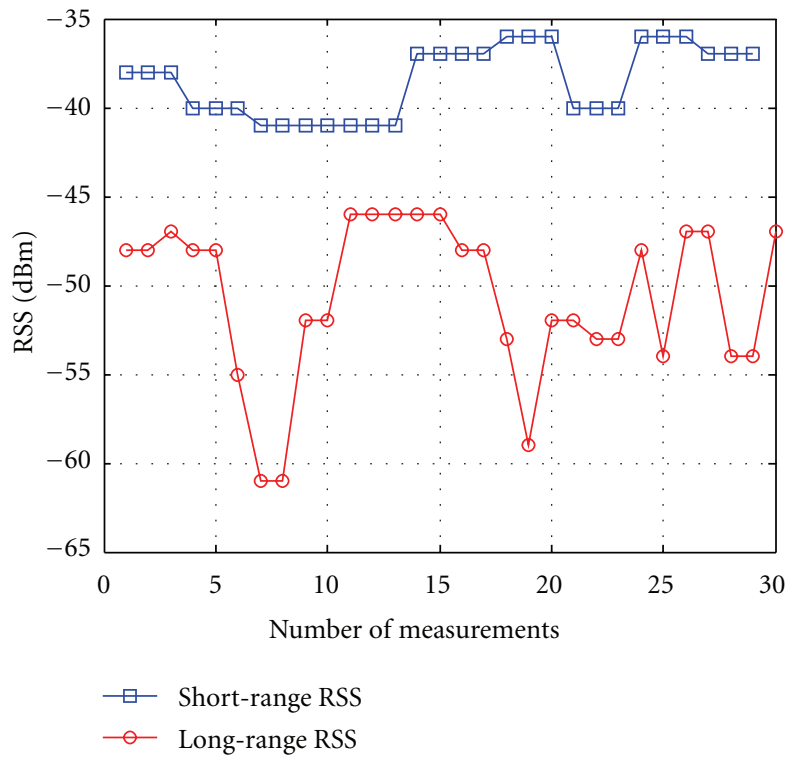

FIGURE 9: Short-range versus long-range measurements.

respectively. The hand grip introduces a loss of approximately $20 \mathrm{dBm}$ in average for the MS under test if compared to the case without the hand impact as shown in Figure 13 (circles) and Figure 14 (circles).

3.6. Effects on Position Estimation. In this experiment we test the effect that the aforementioned human-induced

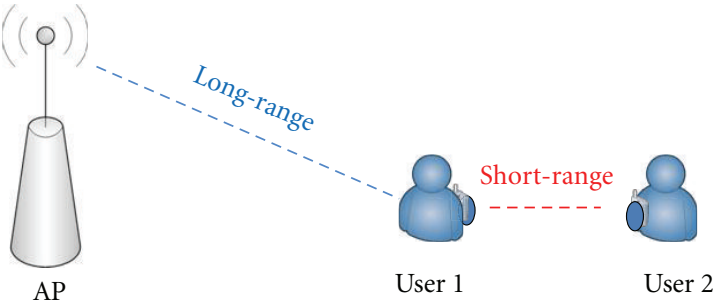

FIGURE 10: Short-range versus long-range scenario.

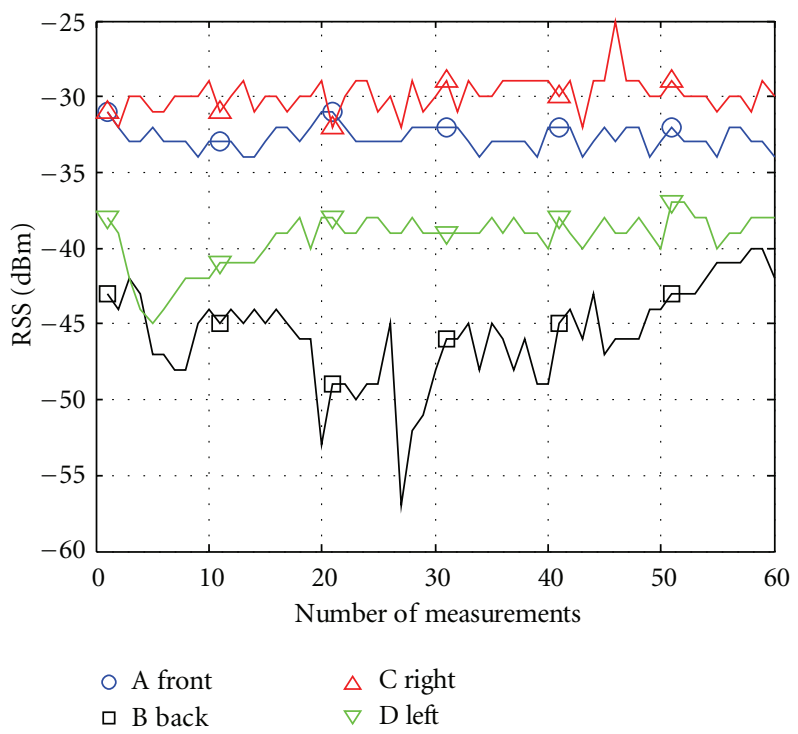

FIGURE 11: Body-loss impact on RSS measurements.

impairments have on the cooperation and hence in the accuracy of the final position estimation. At this purpose, we have devised a squared testing environment using four APs and placing MSs in the center area (Table 1). 


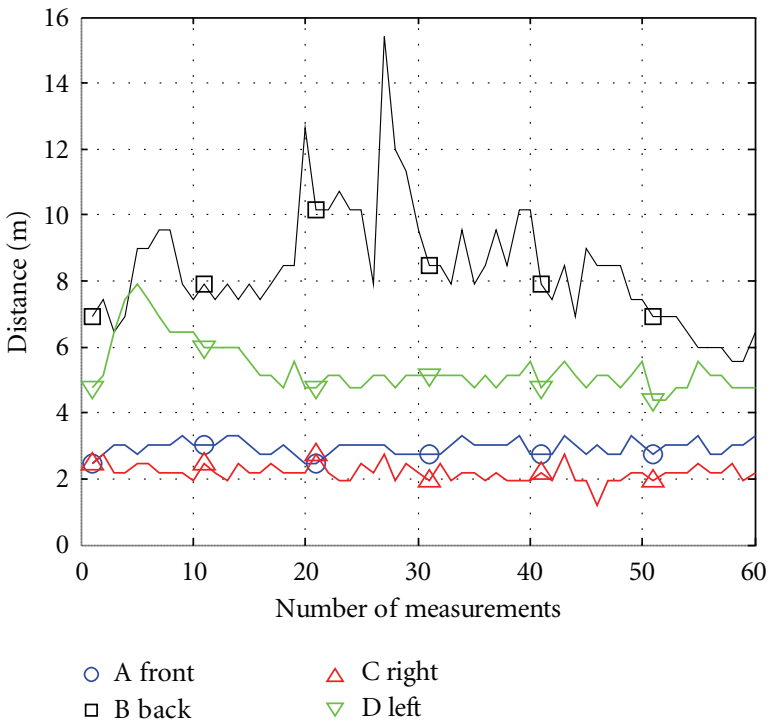

FIgURE 12: Estimated distances with body-loss effect.

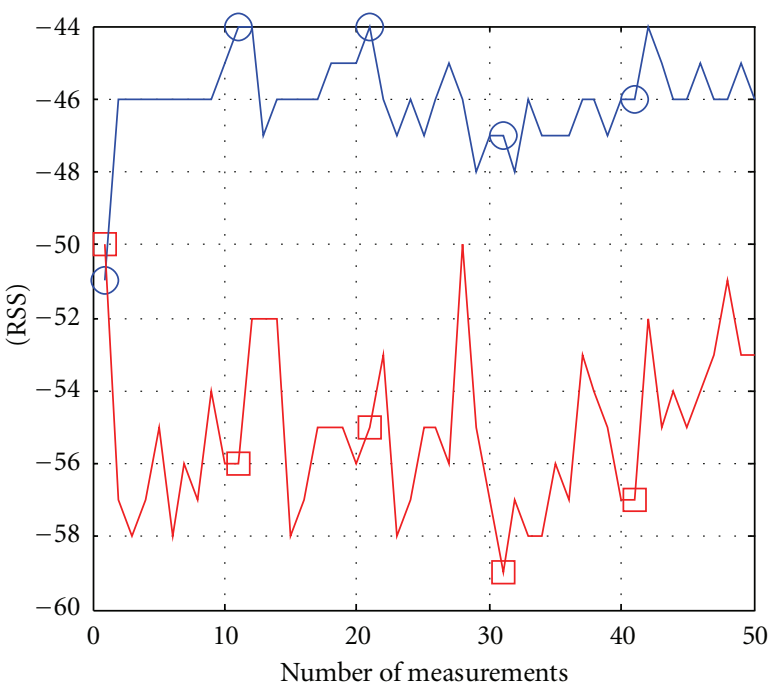

FIGURE 13: Hand-grip impact on RSS measurements.

TABLE 1: Experimental setup.

\begin{tabular}{lcc}
\hline Device & $x(\mathrm{~m})$ & $y(\mathrm{~m})$ \\
\hline AP1 & 0 & 0 \\
AP2 & 0 & 14 \\
AP3 & -7 & 7 \\
AP4 & 7 & 7 \\
MS1 & -1 & 7 \\
MS2 & 1 & 7 \\
MS3 & 0 & 8 \\
\hline
\end{tabular}

A set of 50 RSSs measurements are recorded from each AP by three MSs. Then the empirical path-loss model is applied and the estimated distances from the four APs are sent to the NLLS algorithm (Figure 3(a)). The average of the

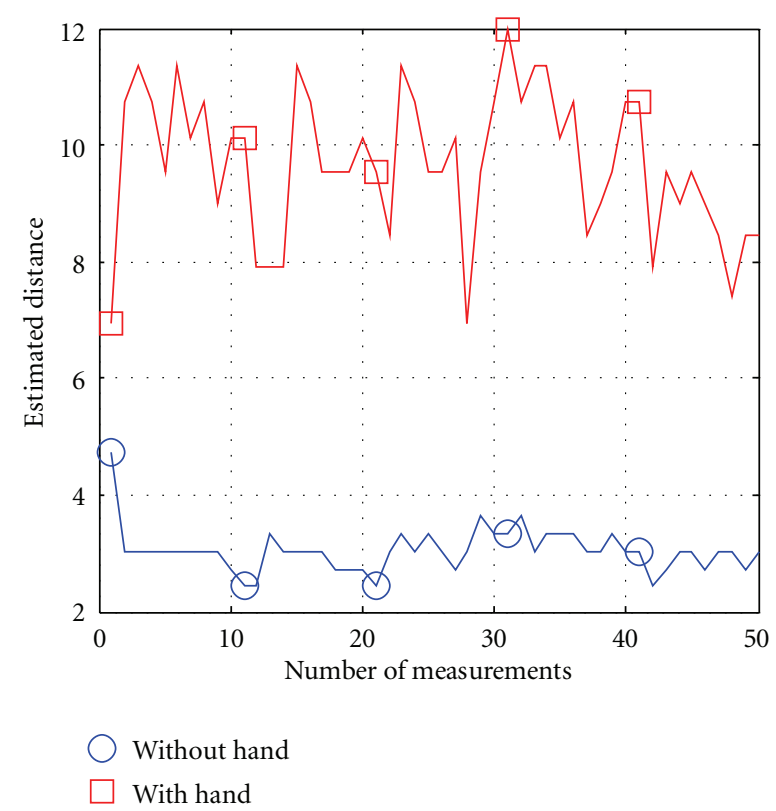

FIGURE 14: Estimated distances with hand-grip effect.

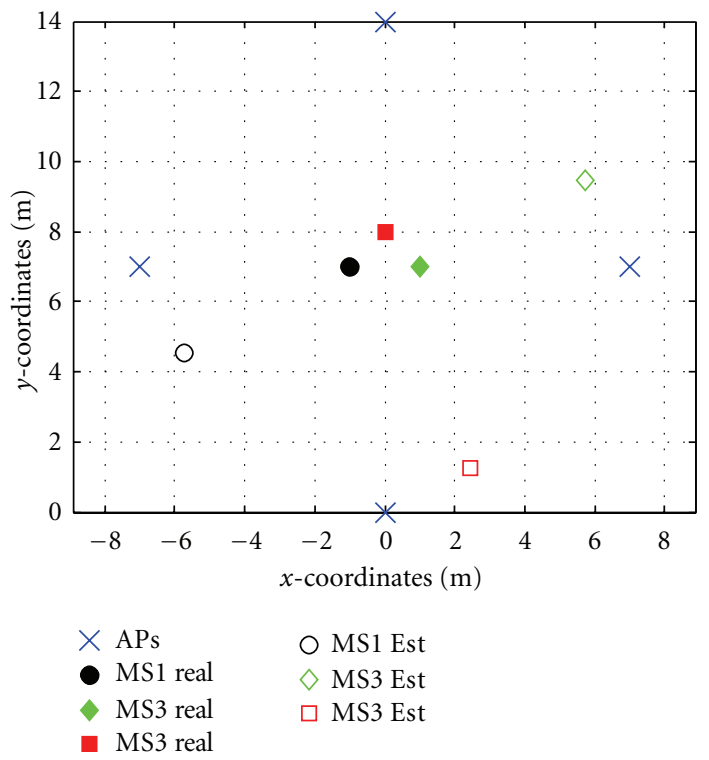

FIGURE 15: Estimated positions without cooperation.

50 estimated positions is shown in Figure 15 showing a clear bias and confirming expected results [3].

3.7. Effects on Range Estimation and Cooperation. Results obtained in Figure 15 could in theory be enhanced by using the proposed data-fusion algorithm as demonstrated in [46]. However, short-distance measurements are corrupted by the influence of the human body and hand-grip as the users are placed like in Figure 18(a), making the proximity estimations very inaccurate so that the data-fusion algorithm underperforms without providing the expected improvements in terms of positioning accuracy (Figure 16). 


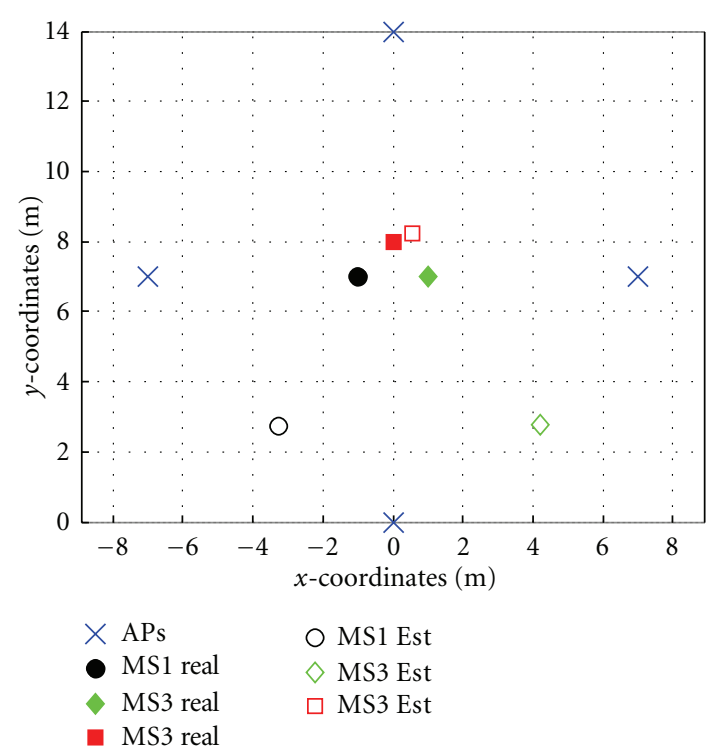

FIGURE 16: Estimated positions with corrupted cooperation.

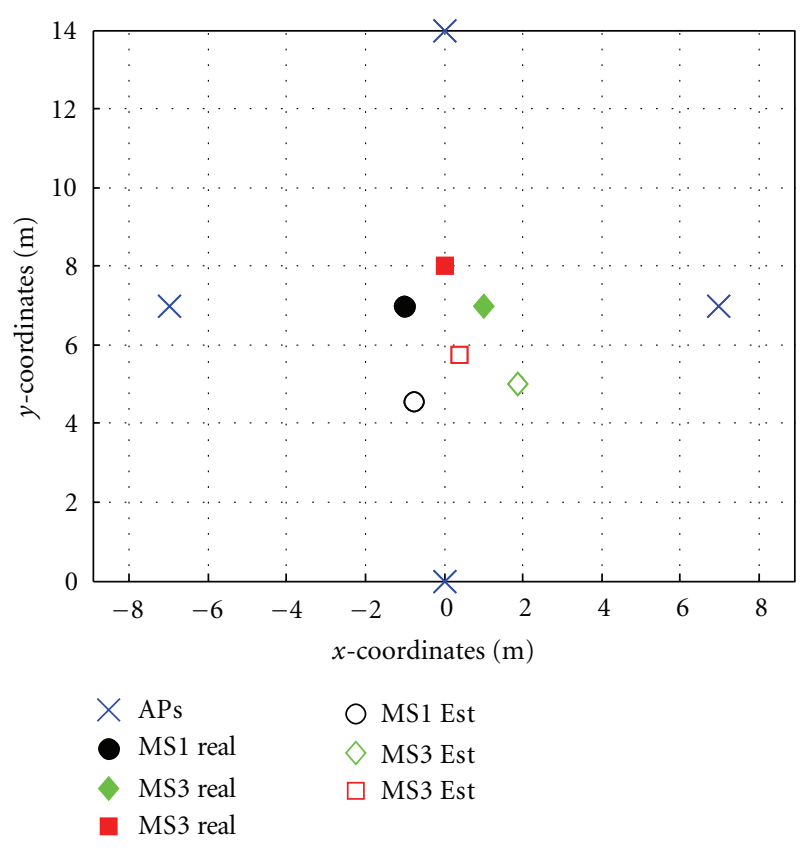

FIGURE 17: Estimated positions with augmented cooperation.

At this point, by knowing the most likely grip effect and orientation of the users among each other (e.g., with the use of an embedded compass) it is possible to apply the correction factors driven by the effect of the human-induced impairments. When applying such corrections to the RSS measured from ad hoc links the cooperation (augmented cooperation) among devices finally has the expected beneficial effect on the overall group positioning accuracy as shown in Figure 17. The overall improvements provided by the cooperation among devices and knowledge of the human impairments are then highlighted in Figures 19 and 20, where

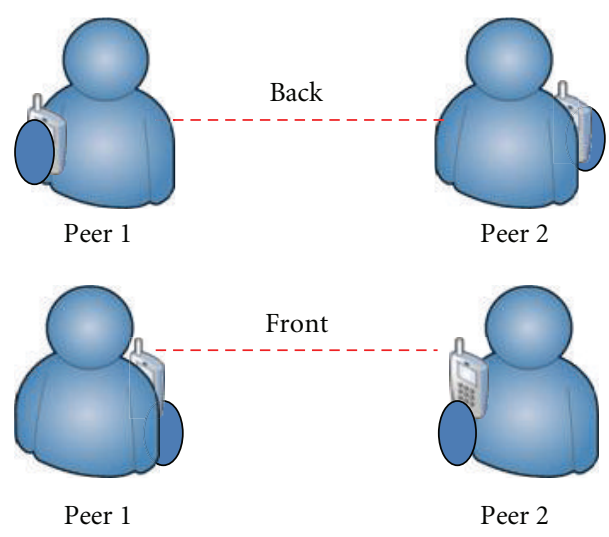

FIGURE 18: User back and front signal blockage.

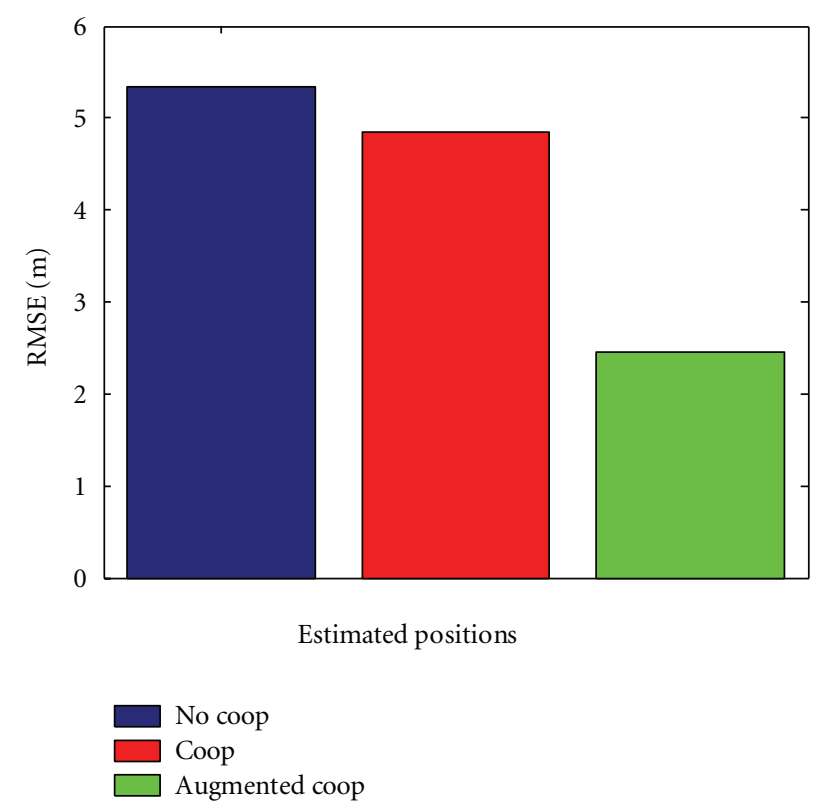

FIGURE 19: RMSE of estimated positions.

the enhancement levels are shown in terms of root meansquared error (RMSE) [5] and percentage gain, respectively.

\section{Conclusions}

In this paper we have first introduced the broad field of wireless positioning for mass market devices, trying to give an overview of the current advancements in literature, highlighting both their potentials and limitations. Reviewing the conventional positioning techniques it has been asserted that they are not able to keep their promise in term of accuracy and feasibility. In fact, indoor environments constitute a highly challenging scenario because of their intrinsic complexity and unpredictability. Even though some attempts have been done to overcome the aforementioned limitations by proposing coarse cooperative techniques on top of conventional algorithms, it has been found that a simplistic modeling of the wireless scenario is not able to capture the intrinsic variability of indoor environments. In fact 


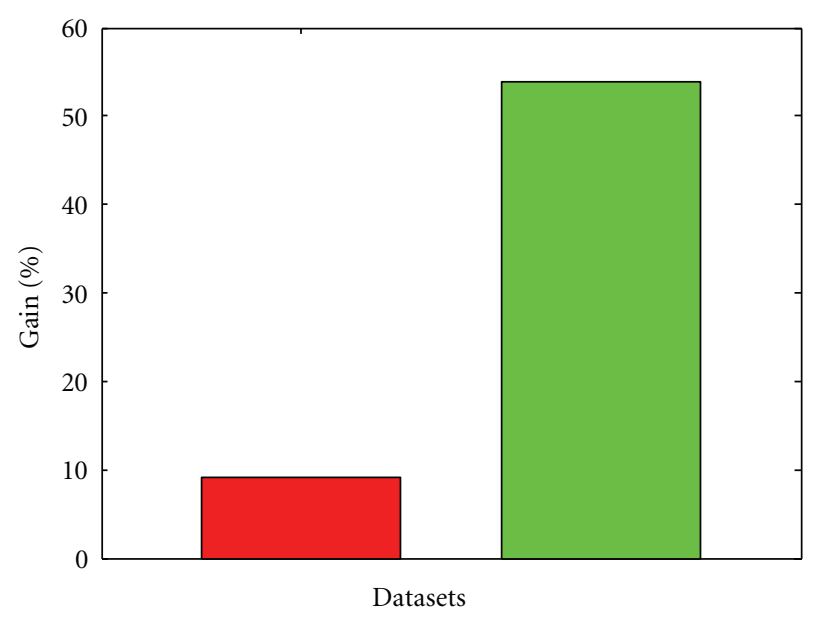

Cooperation

Augmented cooperation

Figure 20: Percentage gain.

the presence of the user's body is an inescapable part in the broader picture of indoor channel modeling. In the past, the user-induced anomalies in the wireless signals have been neglected not only within literature, but also by the very mobile devices manufacturers. At this purpose, in this contribution we have highlighted first and foremost the effect of the user's body on the far-field part of the wireless link, mainly identifying it as a time-varying blocking object. Moreover, the effects of the near-field perturbations caused by the close by environment have been presented. In particular, the influence of the hand-grip on mass market devices has been shown to have a growing importance in mobile positioning algorithms. In this paper, many experimental results have been presented to assess the potential of user's body cognitivity on the cooperative positioning performances. Surprisingly, it has been found that cooperation is not improving significantly the accuracy of the estimated positions of the users with respect to the noncooperative case. In fact, as stated before, the presence of the user should be correctly accounted for in the data-fusion algorithm. This means that if the hand grip and the blocking is somehow accessible to the algorithm we could see a real boost in the cooperative approach. For all these reasons, the encouraging measurement results suggest that future work will highly benefit from a proper knowledge of what surrounds the mobile devices. Additionally, we would like to point out that these benefits could be achieved in a straightforward manner by using off-the-shelf mobile devices that already embed all the needed sensors to unleash cooperative-based positioning services.

\section{Acknowledgments}

The research leading to these results has received funding from the TISE Doctoral Programme, Nokia Foundation,
Ulla Tuomisen säätiö, Tekniikan edistämissäätiön (TES), and Tuula ja Yrjö Neuvo Fund.

\section{References}

[1] F. D. Rosa, H. Leppäkoski, S. Biancullo, and J. Nurmi, "Ad-hoc networks aiding indoor calibrations of heterogeneous devices for fingerprinting applications," in Proceedings of the International Conference on Indoor Positioning and Indoor Navigation (IPIN '10), Zürich, Switzerland, September 2010.

[2] F. D. Rosa, T. Paakki, H. Leppäkoski, and J. Nurmi, "A cooperative framework for path loss calibration and indoor mobile positioning," in Proceedings of the 7th Workshop on Positioning, Navigation and Communication (WPNC '10), pp. 86-92, Dresden, Germany, March 2010.

[3] F. D. Rosa, L. Xu, M. Pelosi, C. Laoudias, A. Terrezza, and J. Nurmi, "Hand-Grip and Body-Loss impact on RSS measurements for localization of mass market devices," in Proceedings of the International Conference on Localization and GNSS (ICLGNSS '11), pp. 58-63, Tampere, Finland, 2011.

[4] J. Figueiras and S. Frattasi, Mobile Positioning and Tracking: From Conventional To Cooperative Techniques, Wiley, New York, NY, USA, 1st edition, 2010.

[5] F. D. Rosa, S. A. Wardana, C. L. F. Mayorga et al., "Experimental activity on cooperative mobile positioning in indoor environments," in Proceedings of the IEEE International Symposium on a World of Wireless, Mobile and Multimedia Networks (WOWMOM '07), Helsinki, Finland, June 2007.

[6] F. D. Rosa, H. Leppkoski, A. Ghalib et al., Ad Hoc Networks For Cooperative Mobile Positioning, IN-TECH, Rijeka, Croatia, 2011.

[7] J. Syrjarinne, Studies on modern techniques for personal positioning [Ph.D. thesis], Tampere University of Technology, Tampere, Finland, 2001.

[8] P. Bahl and V. N. Padmanabhan, "RADAR: an in-building RFbased user location and tracking system," in Proceedings of the 19th Annual Joint Conference of the IEEE Computer and Communications Societies, Reaching the Promised Land of Communications (INFOCOM '00), vol. 2, pp. 775-784, March 2000.

[9] M. Pelosi, O. Franek, M. B. Knudsen, M. Christensen, and G. F. Pedersen, "A grip study for talk and data modes in mobile phones," IEEE Transactions on Antennas and Propagation, vol. 57, no. 4, pp. 856-865, 2009.

[10] F. Harrysson, J. Medbo, A. F. Molisch, A. J. Johansson, and F. Tufvesson, "Efficient experimental evaluation of a MIMO handset with user influence," IEEE Transactions on Wireless Communications, vol. 9, no. 2, pp. 853-863, 2010.

[11] M. Pelosi, M. B. Knudsen, and G. F. Pedersen, "Multiple antenna systems with inherently decoupled radiators," IEEE Transactions on Antennas and Propagation, vol. 60, no. 2, pp. 503-515, 2012.

[12] A. Sibille and A. J. Braga, "Propagation aware statistical modeling of MIMO terminal antennas," in Proceedings of the IEEE International Symposium on Antennas and Propagation and CNC-USNC/URSI Radio Science Meeting, Leading the Wave (AP-S/URSI '10), pp. 1522-3965, July 2010.

[13] A. Sibille and M. A. Mellah, "A statistical model of handsets effective gain accounting for user influence and local propagation," in Proceedings of the 4th European Conference on Antennas and Propagation (EuCAP '10), pp. 1-4, April 2010.

[14] http://kom.aau.dk/ gfp/PhdFinal.pdf, 2012.

[15] J. Holopainen, O. Kivekas, J. Ilvonen, R. Valkonen, C. Icheln, and P. Vainikainen, "Effect of the user's hands on the operation 
of lower UHF-band mobile terminal antennas: focus on digital television receiver," IEEE Transactions on Electromagnetic Compatibility, vol. 53, no. 3, pp. 831-841, 2011.

[16] C. Oliveira and L. M. Correia, "A statistical model to characterize user influence in body area networks," in Proceedings of the 10th COST2100, TD(10)10091, Athens, Greece, February 2010.

[17] M. Pelosi, O. Franek, M. B. Knudsen, and G. F. Pedersen, "Influence of dielectric loading on PIFA antennas in close proximity to user's body," Electronics Letters, vol. 45, no. 5, pp. 246-248, 2009.

[18] C. H. Li, E. Ofli, N. Chavannes, and N. Kuster, "Effects of hand phantom on mobile phone antenna performance," IEEE Transactions on Antennas and Propagation, vol. 57, no. 9, pp. 2763 2770, 2009.

[19] G. F. Pederen, J. Ø. Nielsen, O. Franek, J. B. Andersen, M. Pelosi, and Y. Wang, "Measurement based investigations for future communication system performance evaluation," in Proceedings of the Loughborough Antennas and Propagation Conference (LAPC '09), pp. 23-30, November 2009.

[20] CTIA Certication, Test Plan For Mobile Station Over the Air PerFormance, CTIA Wireless Association, Washington, DC, USA, 2005.

[21] http://kn.theiet.org/magazine/eletters/4603/helping-hands.cfm.

[22] M. Pelosi, O. Franek, M. B. Knudsen, G. F. Pedersen, and J. B. Andersen, "Antenna proximity effects for talk and data modes in mobile phones," IEEE Antennas and Propagation Magazine, vol. 52, no. 3, pp. 15-27, 2010.

[23] P. Vainikainen, J. Holopainen, C. Icheln et al., "More than 20 antenna elements in future mobile phones, threat or opportunity?" in Proceedings of the 3rd European Conference on Antennas and Propagation (EuCAP'09), pp. 2940-2943, March 2009.

[24] S. Myllymaki, A. Huttunen, M. Berg, M. Komulainen, and H. Jantunen, "Method for measuring user-induced load on mobile terminal antenna," Electronics Letters, vol. 45, no. 21, pp. 1065-1066, 2009.

[25] A. Huttunen, S. Myllymaki, M. Komulainen, and H. Jantunen, "Capacitive sensor arrangement to detect external load on a mobile terminal antenna," Progress In Electromagnetics Research Letters, vol. 15, pp. 13-18, 2010.

[26] W. Chang, K. E. Kim, H. Lee et al., "Recognition CHI, 2009, techniques for mobile interaction," in Proceedings of the 27th International Conference on Human Factors in Computing Systems (CHI '09), Boston, MA, USA, April 2009.

[27] F. Harrysson, J. Medbo, A. Derneryd, and A. Molisch, "Performance of a MIMO terminal including a user phantom in a stationary micro-cell scenario with comparison between a ray-based method and direct measurements," Tech. Rep. TD07-379, PIB Publisher, Duisburg, Germany, 2007.

[28] F. Harrysson, J. Medbo, and A. Molisch, "Indoor performance of a MIMO handset including user influence by comparing a composite channel method with direct measurements," Tech. Rep. TD-08-661, DocPresse ESJ, Lille, France, 2008.

[29] F. Harrysson, J. Medbo, A. F. Molisch, A. J. Johansson, and F. Tufvesson, "Efficient experimental evaluation of a MIMO handset with user influence," IEEE Transactions on Wireless Communications, vol. 9, no. 2, pp. 853-863, 2010.

[30] K. Kaemarungsi and P. Krishnamurthy, "Properties of indoor received signal strength for WLAN location fingerprinting," in Proceedings of the 1st Annual International Conference on Mobile and Ubiquitous Systems: Networking and Services (MOBIQUITOUS '04), pp. 14-23, August 2004.
[31] T. King, S. Kopf, T. Haenselmann, C. Lubberger, and W. Effelsberg, "COMPASS: a probabilistic indoor positioning system based on 802.11 and digital compasses," in Proceedings of the 1st ACM International Workshop on Wireless Network Testbeds, Experimental Evaluation and Characterization (WiNTECH '06), pp. 34-40, September 2006.

[32] P. Bahl, V. Padmanabhan, and A. Balachandran, "Enhancements to the RADAR user location and tracking system, Microsoft Research,” Tech. Rep. MSR-TR-00-12, 2000.

[33] A. M. Ladd, K. E. Bekris, A. Rudys, L. E. Kavraki, and D. S. Wallach, "Robotics-based location sensing using wireless ethernet," Wireless Networks, vol. 11, no. 1-2, pp. 189-204, 2005.

[34] Y. Gu, A. Lo, and I. Niemegeers, "A survey of indoor positioning systems for wireless personal networks," IEEE Communications Surveys and Tutorials, vol. 11, no. 1, pp. 13-32, 2009.

[35] Y. Wang, X. Jia, and H. K. Lee, "An Indoor wireless positioning system based on wireless local area network infrastructure," in Proceedings of the 6th International Symposium on Satellite Navigation Technology Including Mobile Positioning and Location Services, 2003.

[36] E. C. L. Chan, G. Baciu, and S. C. Mak, "Orientation-based Wi-Fi positioning on the Google nexus one," in Proceedings of the 6th Annual IEEE International Conference on Wireless and Mobile Computing, Networking and Communications (WiMob '10), pp. 392-397, October 2010.

[37] M. I. Mackowiak, Correia, and M. Luis, "Towards a radio channel model for off-body communications in a multipath environment," in Proceedings of the 18th European Wireless Conference (EW'12), pp. 1-7, April 2012. 

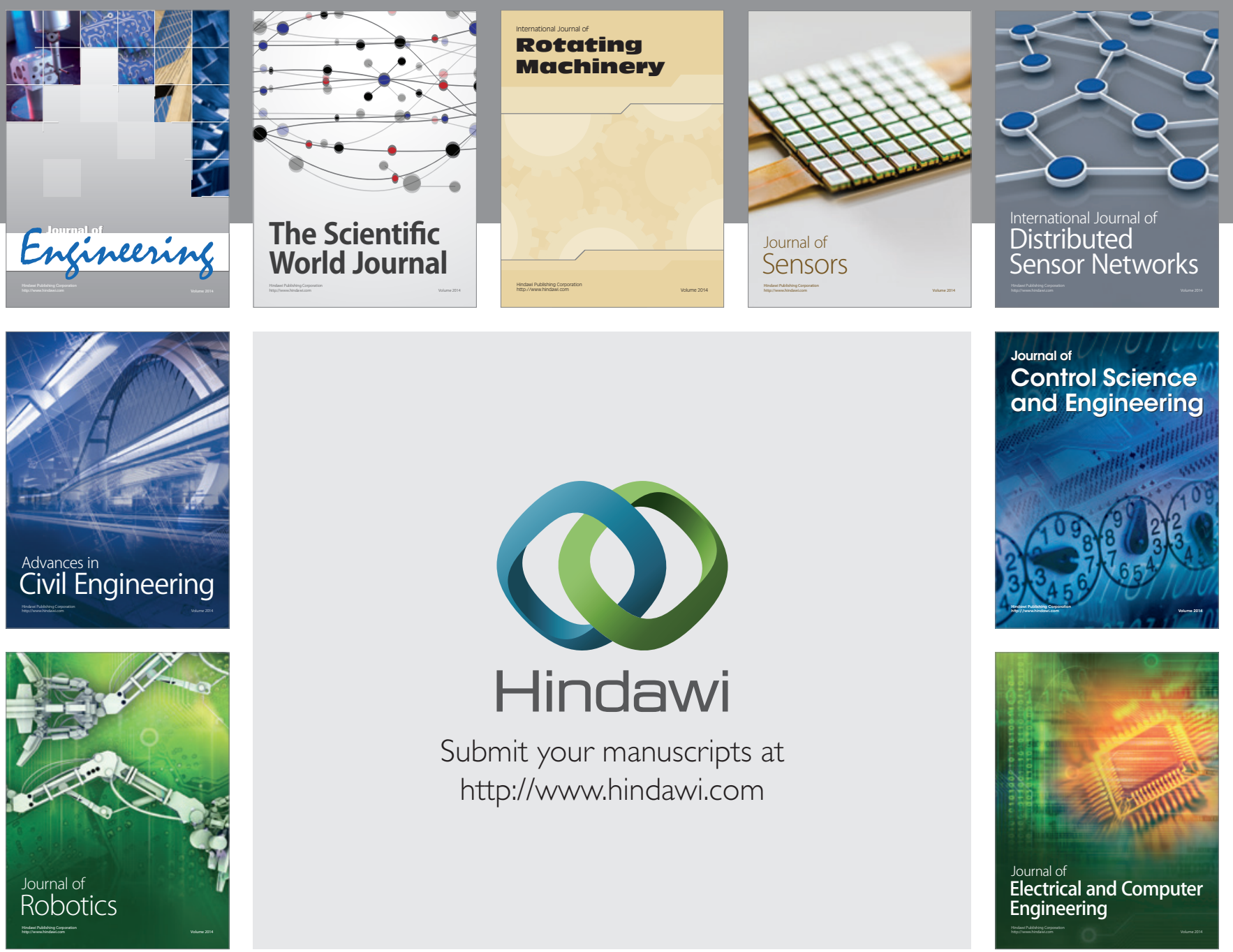

Submit your manuscripts at

http://www.hindawi.com
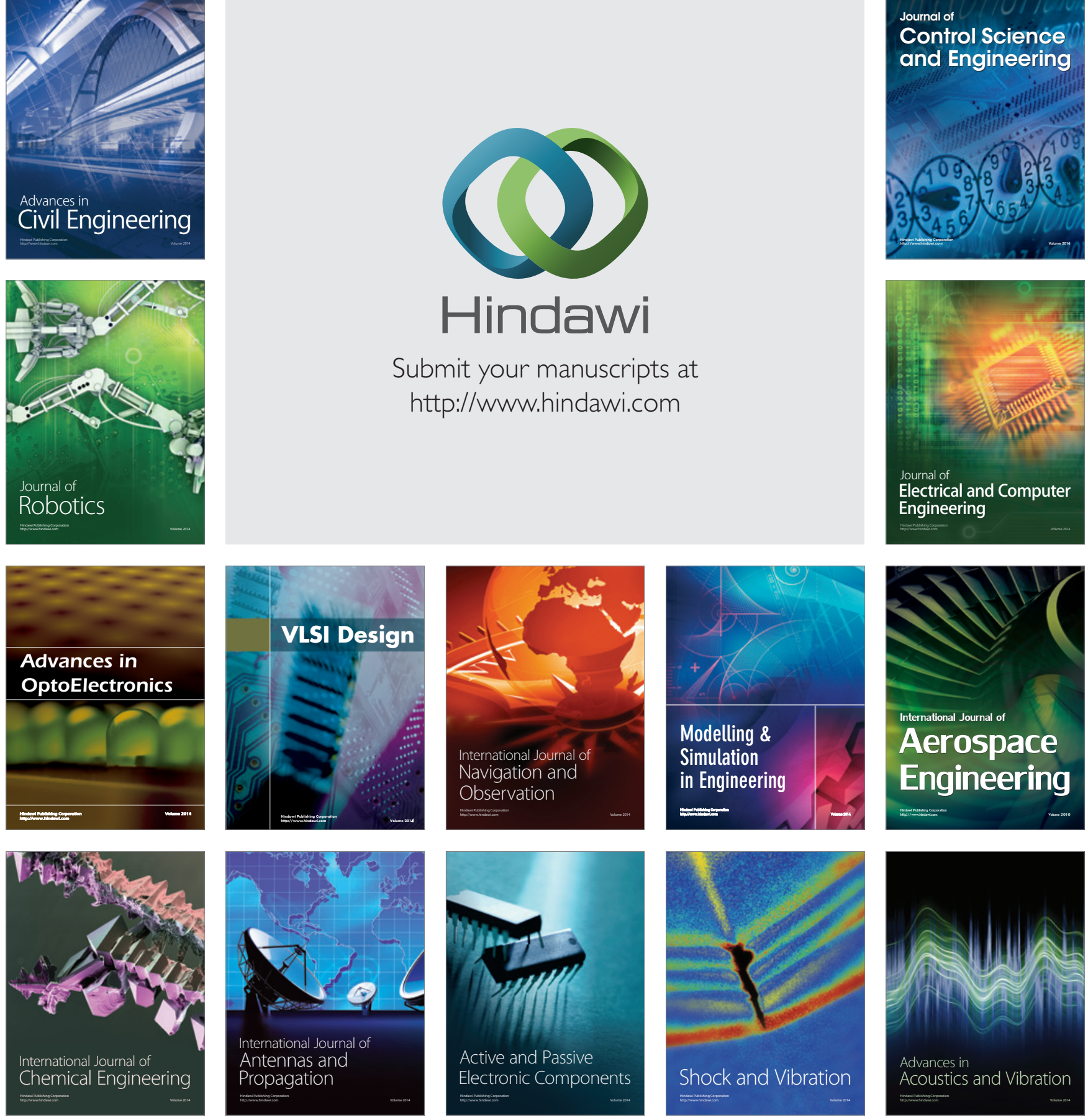